\title{
Early abnormalities of pisiform and triquetrum in rheumatoid arthritis
}

\author{
DONALD RESNICK \\ From the Department of Radiology, Veterans Administration Hospital, San Diego, and \\ University Hospital, University of California, San Diego
}

\begin{abstract}
Resnick, D. (1976). Annals of the Rheumatic Diseases, 35, 46-50. Early abnormalities of pisiform and triquetrum in rheumatoid arthritis. Erosions on the triquetrum and pisiform are frequent in early rheumatoid arthritis and occur characteristically at 3 sites. (a) A shallow erosion on the proximal medial triquetral 'bare' area is related to synovial proliferation at the margin of the radiocarpal cavity, although compressive forces from an adjacent long ulnar styloid may be contributory; (b) an erosive abnormality on the distal medial triquetrum is related to synovial proliferation along the margin of the midcarpal joint ; and (c) ring-like erosions on apposing surfaces of the triquetrum and pisiform are produced by pannus within the pisiform-triquetral compartment.
\end{abstract}

Bone erosions in the rheumatoid wrist have been classified (Martel, Hayes, and Duff, 1965) as marginal erosions occurring near the capsular attachments, compression erosions occurring at sites of muscular forces acting on deossified bone, and superficial surface resorption, perhaps related to adjacent tenosynovitis. The pathogenesis of early radiographic abnormalities about the ulnar styloid has been discussed (Resnick, 1974b), as have characteristic alterations in the triquetrum (Martel and others, 1965; Norgaard, 1969). In order to define better the mechanism and incidence of triquetral and pisiform erosions in early rheumatoid arthritis (RA), a comprehensive study of the rheumatoid wrist was undertaken. Material included (a) $x$-rays of 100 patients with classical or definite RA by American

FIG. 1 Radiocarpal and midcarpal compartment anatomy. A coronal section through the wrist outlines the radiocarpal compartment (1) separated from the inferior radioulnar compartment (2) by the triangular fibrocartilage (4). A meniscus-homologue (5) attaches to the triquetrum (TRIQ) creating a $Y$-shaped ulnar limit to the radiocarpal compartment; the proximal prestyloid diverticulum (3) is poorly identified but the distal continuation of the radiocarpal joint (A) extends around a portion of the triquetrum. The midcarpal compartment (6), separated from the radiocarpal compartment by interosseous ligaments (8) between bones of the proximal carpal row, has an ulnar limit intimate with the distal triquetrum $(B)$. A portion of the carpometacarpal joint (7) is seen

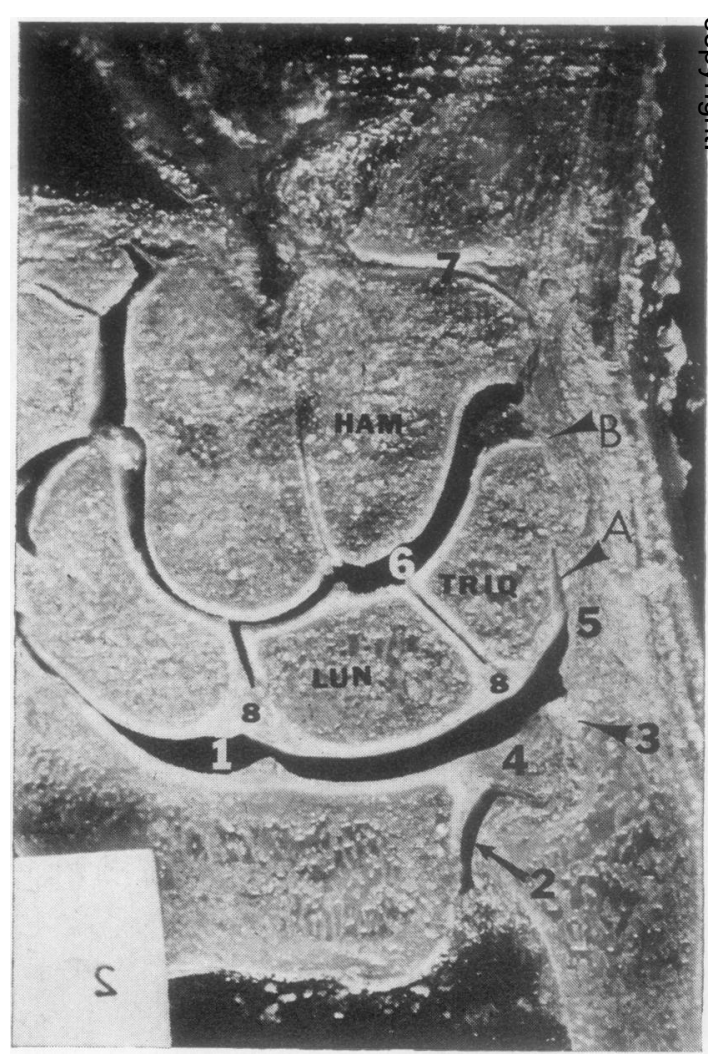

Accepted for publication June 16, 1975 .

Address of author: Veterans Administration Hospital, 3350 La Jolla Village Drive, San Diego, Ca. 92161. 


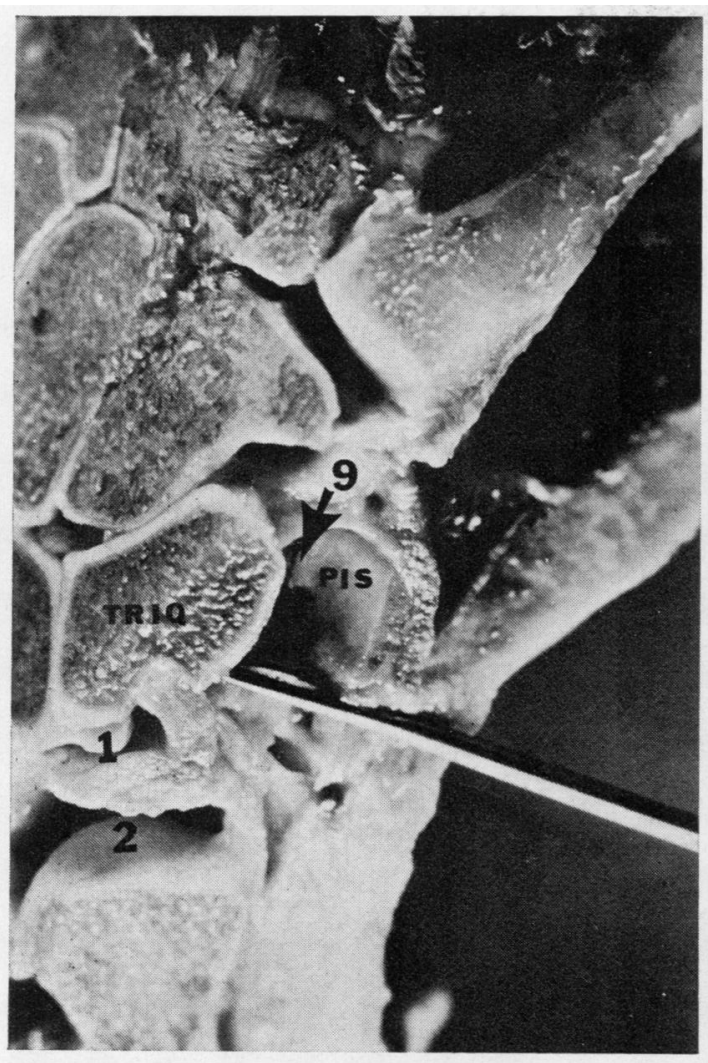

A

FIG. 2 Pisiform-triquetral compartment anatomy. (A) A coronal section outlines distinct radiocarpal (1), inferior radioulnar (2), and pisiform-triquetral (9) compartments. (B) Forceful contrast injection into the pisiform-triquetral compartment shows its continuity with the radiocarpal joint (I). (C) A sagittal section of another cadaveric wrist shows the extent of the pisiform-triquetral compartment (9). Note the large proximal (curved arrow) and smaller distal (straight arrow) extensions

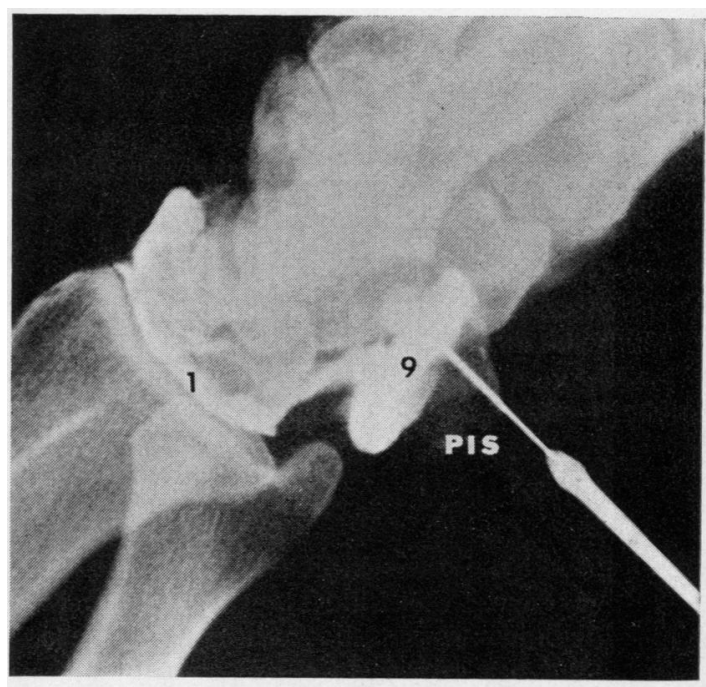

$B$

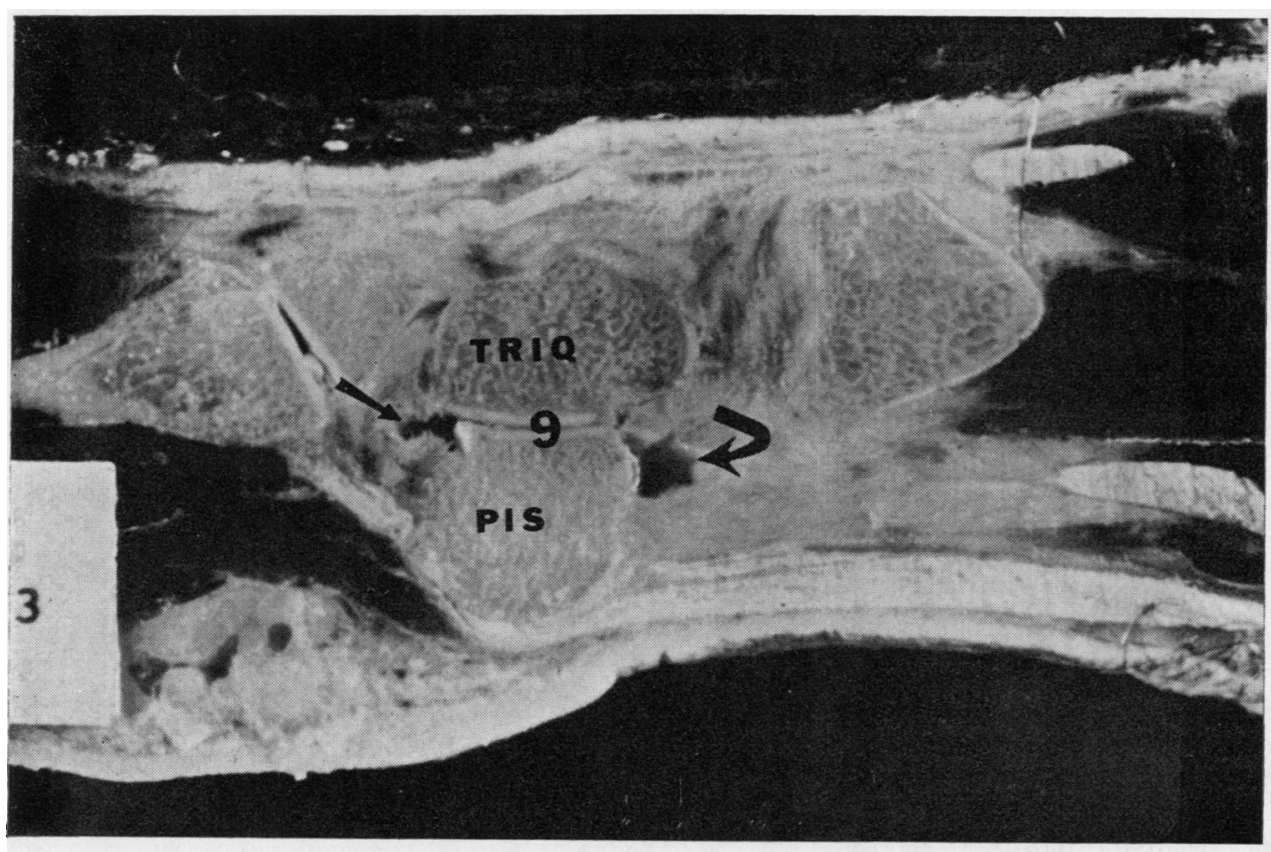

C 
Rheumatism Association criteria, (b) wrist arthrograms in 40 rheumatoid patients, and in 6 rheumatoid and 20 'control' cadavers, and (c) gross and microscopical sections from 6 rheumatoid cadavers. The microscopical preparation from slabs $1-3 \mathrm{~cm}$ thick included decalcification in formic acid and paraffin embedding. Sections 8-10 $\mu \mathrm{m}$ thick were then obtained and stained with haematoxylin and eosin.

\section{Pertinent anatomy}

Previous articles have discussed in depth the compartmental anatomy of the wrist (Resnick, 1974a,b), therefore features pertinent to the triquetrum and pisiform only will be discussed.

\section{RADIOCARPAL COMPARTMENT}

The ulnar limit of the radiocarpal joint is created in most individuals by the firm attachment of the meniscus-homologue to the triquetrum (Lewis, Hamshere, and Bucknill, 1970)(Fig. 1). Thus, the Y-shaped medial end of the radiocarpal compartment contains two 'diverticuli'; the prestyloid recess, the more proximal limb, approaches the ulnar styloid whereas a distal limb is intimate with the proximal radial $2 / 3$ of the triquetrum. Much of the latter bone is related to the joint capsule; a strong attachment between the distal ulna and the proximal carpal row is best termed the ulnatriquetral band of the ulnar collateral ligament (Lewis and others, 1970). The synovial membrane adjacent to this ligament reflects over a 'bare' area of the triquetrum, i.e. a portion of the bone lacking a significant cartilage-coat (Martel and others, 1965).

\section{MIDCARPAL COMPARTMENT}

This articular cavity separates the proximal and distal carpal rows (Fig. 1). On its ulnar limit the joint may widen creating a small space between the triquetrum and hamate. In this area the synovium extends over the distal medial triquetrum adjacent to the capsule.

\section{PISIFORM-TRIQUETRAL COMPARTMENT}

Although anatomical dissections may show radiocarpal-pisiform-triquetral joint communication in $34 \%$ of cadavers (Lewis and others, 1970), more frequently the latter exists as a distinct cavity (Fig. $2 A$ ). Arthrographic studies confirm the integrity of the joint capsule in this area, although forceful injection of contrast into either radiocarpal or pisiformtriquetral compartment may outline their communication (Fig. 2B). The pisiform-triquetral joint is a synovial joint with a loose fibrous articular capsule attached to the pisiform and triquetral bones adjacent to their articular surfaces (Fig. 2C). Arthrography shows a variable proximal extension or recess which may relate to ballooning of the synovial membrane produced by the normal distal migration of the pisiform during fetal development (Lewis, 1971). The contrast-filled joint produces in the sagittal plane a smooth linear shadow between the two bones with a small distal and larger proximal recess. In the coronal plane it is circular or ring-like in appearance.

\section{Pertinent pathological alterations}

The early erosions of the pisiform and triquetrum in RA result from abnormalities in 3 areas: (a) the medial margin of the radiocarpal joint, (b) the medial margin of the midcarpal joint, and (c) the pisiformtriquetral joint.

(a) The characteristic alteration on the proximal medial margin of the triquetrum represents a marginal erosion occurring on the 'bare' area of that bone (Fig. $3)$. A small surface irregularity with cortical loss and subjacent demineralization may later become a deep erosion. That compressive forces may exaggerate this early abnormality is well recognized (Martel and others, 1965). In normal individuals and RA patients a long ulnar styloid may contact the triquetrum in

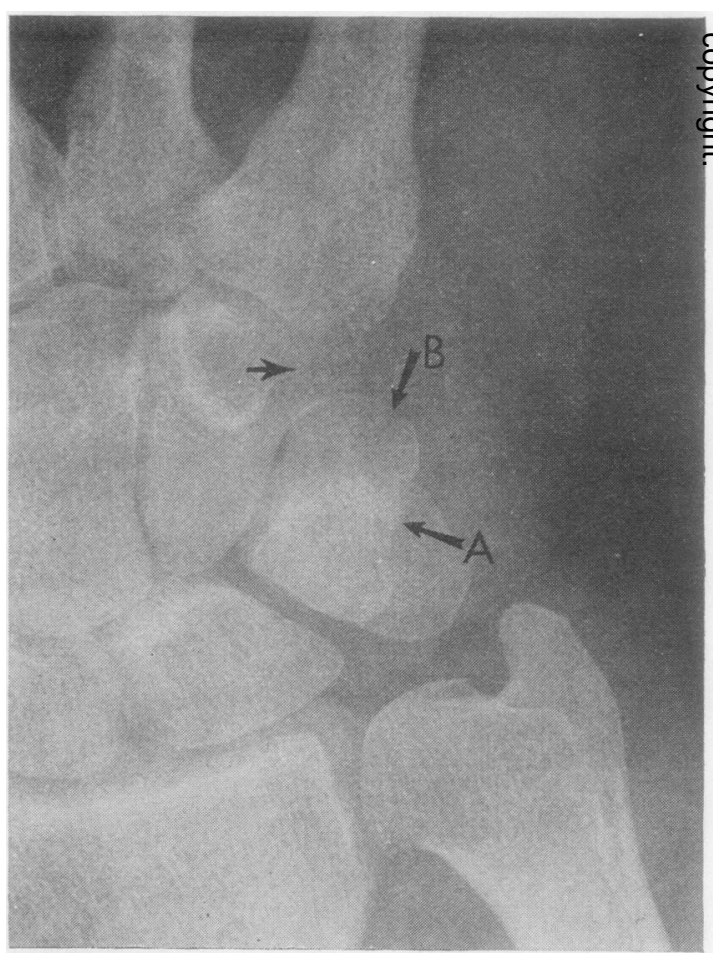

FIG. 3 Sites of triquetral marginal erosions. Erosions related to the ulnar limits of the radiocarpal $(A)$ and midcarpal $(B)$ compartments are apparent in a patient with $R A$. Additional erosive abnormalities of the medial hamate can be seen (arrow) 


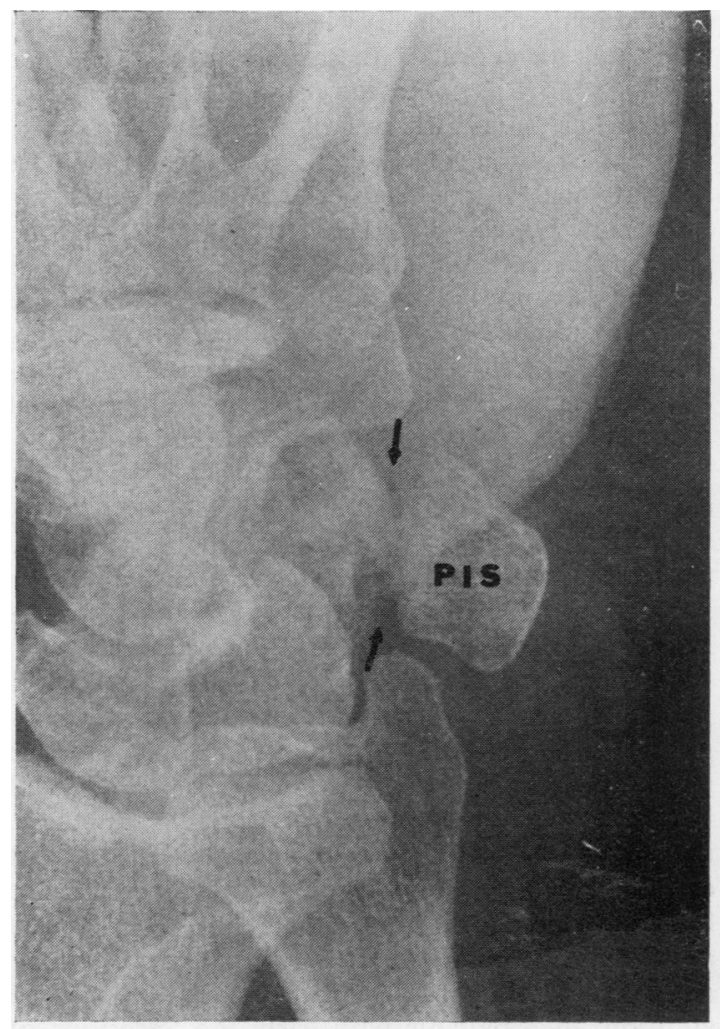

A

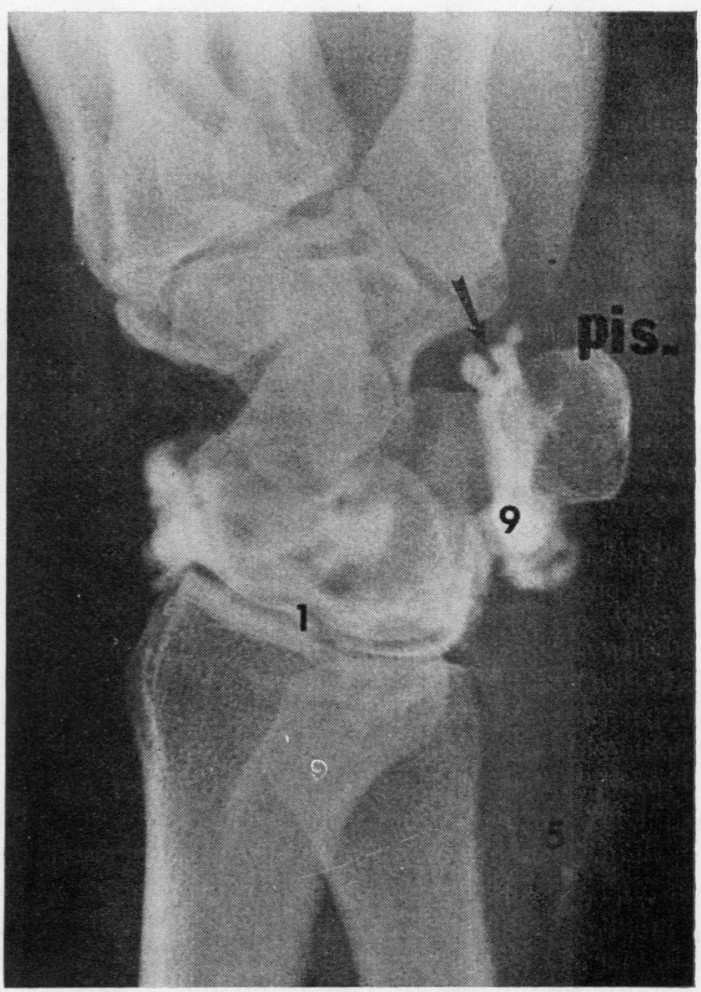

B

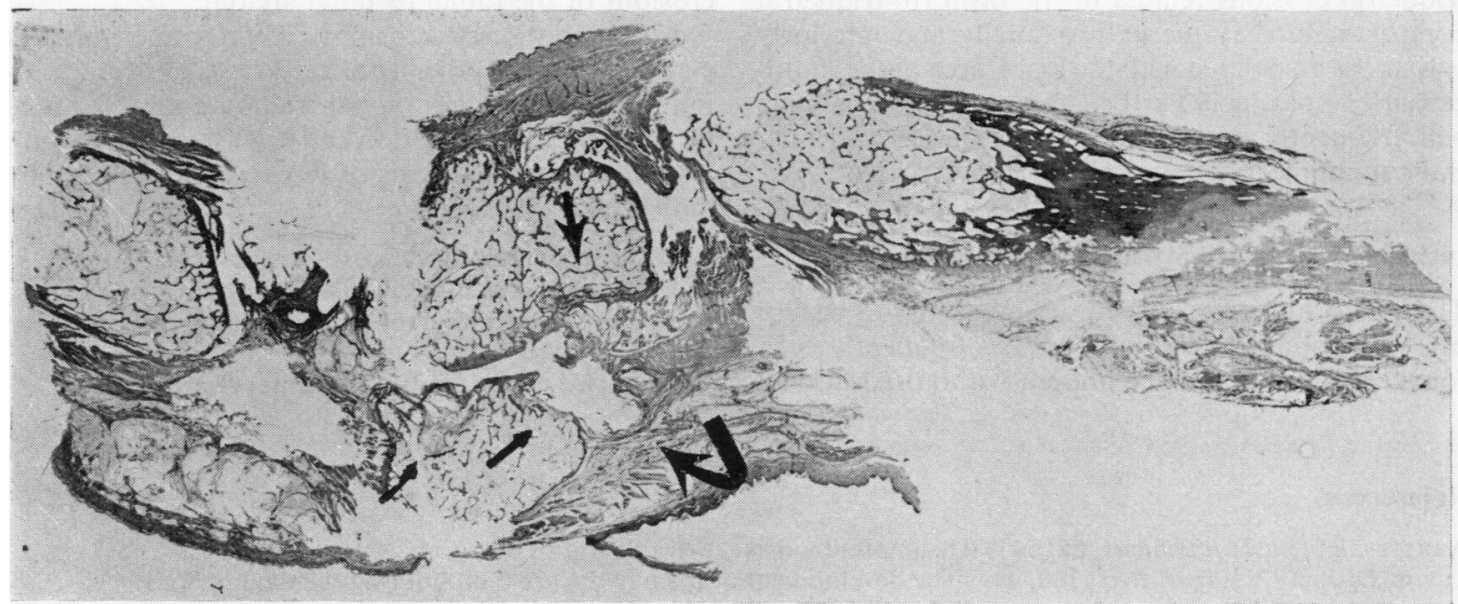

C

FIG. 4 Pisiform-triquetral compartment abnormalities. (A) Ring-like erosions (arrows) on the dorsal pisiform and adjacent triquetrum are related to synovial proliferation in the pisiform-triquetral compartment. (B) Wrist arthrogram in an $R A$ patient outlines communication of the radiocarpal (1) and pisiform-triquetral (9) compartments. At the latter site the synovium (arrow) is irregular and lymphatics (5) can be seen. $(C)$ A photomicrograph $(\times 2)$ of a sagittal section of a rheumatoid wrist outlines synovial proliferation (curved arrows) within the pisiform-triquetral compartment creating extensive erosions (straight arrows) on apposing surfaces of triquetrum and pisiform 
ulnar deviation at the wrist via the opening of the prestyloid recess (Lewis and others, 1970). The triquetrum, with cartilage and cortex already compromised by rheumatoid pannus, would be easily victimized by this repeated trauma. In chronic RA, sclerosis and fragmentation of adjacent surfaces of triquetrum and ulnar styloid are frequent (Arkless, 1967; Resnick and Gmelich, 1975).

(b) Equally distinctive are early rheumatoid erosions of the distal surface of the triquetrum adjacent to the medial capsular attachment of the midcarpal joint. In this 'bare' area cortical loss, surface irregularity, and focal demineralization are noted, and associated erosive changes on the hamate are usually seen (Fig. 3). An irregular pool of contrast material between the hamate and triquetrum at the medial margin of the midcarpal compartment during arthrography is characteristic of the rheumatoid wrist.

(c) Synovial proliferation within the pisiformtriquetral compartment may produce early and characteristic $x$-ray abnormality. An $x$-ray taken in the 'reverse' oblique or semisupinated position is necessary for adequate evaluation (Norgaard, 1969). Small erosions are noted on the dorsal surface of the pisiform, particularly at its distal and proximal margins (Fig. $4 A, B$ ); the midportion of this dorsal aspect may be spared. The location of these early alterations is intimately associated with rheumatoid pannus within the proximal and distal recesses of the joint (Fig. 4C). Adjacent erosive changes on the volar surface of the triquetrum are apparent. The triquetral erosions related to the pisiform-triquetral compartment may merge proximally and inferiorly with those associated with the 'bare' area of the radiocarpal compartment; arthrography in these patients will frequently outline radiocarpal-pisiform-triquetral communication (Fig. 4B).

\section{Incidence and specificity of $x$-ray alterations}

The pisiform and triquetrum were chosen because early erosion of these two bones has frequently been found in RA and may be present without additional radiographic abnormality. In a retrospective study the exact incidence of these defects is difficult to determine for the following reasons. (a) On routine (and hence limited) views of the hand and wrist resulting overlap of osseous structures precludes accurate analysis of these erosions in many cases. In particular, evaluation of the pisiform-triquetral joint necessitates 'reverse' oblique positioning, which was obtained in only 88 wrists in this series. (b) In the patients studied, many of whom had long-standing disease, joint space narrowing, sclerosis, and subluxation frequently obscured or obliterated the site of early erosion.

Erosions of the triquetrum at the site of the radiocarpal 'bare' area occurred in $38 \%$ of adequately examined rheumatoid wrists, at the site of the midcarpal 'bare' area in $23 \%$, and along apposing surfaces of pisiform and triquetrum in $41 \%$. The incidence of erosion at the latter location may seem to be unusually high but reflects a smaller number of patients with complete (and high quality) radiographic examinations of the wrist in whom the pisiform-triquetral joint could be evaluated, and hence the interest in this joint cavity. Thus, the incidence of erosive changes at these sites is not dissimilar from that noted at other characteristio locations in the wrist, particularly the ulnar styloie or adjacent groove $(46 \%)$ and mid-portion of the navicular (36\%) (Martel and others, 1965). In addition, in 5 patients with early RA, erosions of the triquetrum and/or pisiform were present without radiocarpal or midcarpal joint space narrowing or erosions of the radial or ulnar styloid.

Although discrete erosions on the triquetrum and pisiform occur in other arthritides (e.g. gout, ankylosing spondylitis), additional radiographic features including demineralization and joint space narrowing allow a correct diagnosis of RA to be made in most individuals.

I gratefully acknowledge the assistance of Mary Gonsalves and Peggy Mackey in preparing the histological sections, Sue Brown for photography, and Janet Zatlokowicz for secretarial help.

Supported in part by V.A. Hospital Grant 7406.

\section{References}

ARKLESS, R. (1967) Radiology, 88, 543 (Rheumatoid wrists: cineradiography)

LEWIS, O. J. (1971) Anat. Rec., 166, 499 (The development of the human wrist joint during the fetal period)

- Hamshere, R. J., ANd Bucknill, T. M. (1970) J. Anat., 106, 539 (The anatomy of the wrist joint)

MARTel, W., HAYeS, J. T., AND Duff, I. F. (1965) Radiology, 84, 204 (The pattern of bone erosion in the hand and wrist in rheumatoid arthritis)

NorgaARD, F. (1969) Ibid., 92, 299 (Earliest roentgen changes in polyarthritis of the rheumatoid type. Continued investigations)

RESNICK, D. (1974a) Ibid., 113, 331 (Arthrography in the evaluation of arthritic disorders of the wrist)

- (1974b) Ibid., 112, 29 (Rheumatoid arthritis of the wrist: why the ulnar styloid?)

—, AND GMELICH, J. (1975) Ibid., 114, 315 (Bone fragmentation in the rheumatoid wrist: radiographic and pathologic considerations) 